\title{
Pulmonary Hamartoma Diagnosed by Convex Probe Endobronchial Ultrasound-Guided Transbronchial Needle Aspiration (EBUS-TBNA)
}

\author{
Yoshinobu Hata ${ }^{1}$, Kazutoshi Isobe ${ }^{2}$, Shuichi Sasamoto ${ }^{1}$, Kazuyoshi Tamaki ${ }^{1}$, \\ Shoji Takahashi ${ }^{1}$, Fumitomo Sato ${ }^{1}$, Aki Mitsuda ${ }^{3}$, Yoichiro Okubo ${ }^{3}$, Kazutoshi Shibuya ${ }^{3}$, \\ Sakae Homma ${ }^{2}$ and Keigo Takagi ${ }^{1}$
}

\begin{abstract}
A 71-year-old man with a right hilar mass shadow was referred to our department. Chest computed tomography showed a small fatty area inside. Convex probe endobronchial ultrasound (CP-EBUS) images revealed a heterogeneous low-echoic mass, resembling a stone wall and showing a smooth sliding movement along the tracheal wall during respiration. Transbronchial needle aspiration (EBUS-TBNA) was performed and cartilage cells were identified, compatible with the presence of a hamartoma. The patient requested follow-up without surgical intervention. CP-EBUS images are easier to interpret than other methods for monitoring respiratory dynamics. We conclude that CP-EBUS is a useful addition to techniques both for noninvasive imaging as well as guiding pathological examination.
\end{abstract}

Key words: EBUS-TBNA, hamartoma

(Inter Med 49: 1171-1173, 2010)

(DOI: 10.2169/internalmedicine.49.3219)

\section{Introduction}

Convex probe endobronchial ultrasound (CP-EBUS) is a convex transducer at the tip of a flexible bronchoscope, which scans parallel to the direction of insertion of the bronchoscope (1). The resulting linear ultrasound images are relatively easy to interpret, enabling monitoring of respiratory dynamics, and possibly providing a useful tool for evaluating mediastinal invasion of intrapulmonary lesions. Here, we report a case of intrapulmonary hamartoma diagnosed by CP-EBUS examination and EBUS-transbronchial needle aspiration (EBUS-TBNA).

\section{Case Report}

A 71-year-old man was referred to our department for work-up of a right hilar mass shadow in November 2008
(Fig. 1A). The patient had quit smoking 20 years previously with a smoking history of 15 pack years. Chest computed tomography (CT) showed a mass shadow at the right hilum adjacent to the trachea and superior vena cava, which was $3.5 \mathrm{~cm}$ in the diameter. Thin-slice CT revealed a small fatty area in the tumor (Fig. 1B). Although intrapulmonary hamartoma was the most probable diagnosis, lung cancer invading the mediastinum, mediastinal tumor or metastatic lymph node could not be excluded. F18-fluorodeoxyglucose positron emission tomography (FDG-PET) showed no abnormal accumulation in the tumor or any other site. $\mathrm{CP}$ EBUS was performed to determine the nature of the mass (BF-UC260F-OL8, Olympus). CP-EBUS images revealed a heterogeneous low-echoic mass consisting of high-echoic dividing lines and coarse spots inside, resembling a stone wall (Fig. 2). The tumor showed a smooth sliding movement along the tracheal wall during respiration, which indicated that the lesion was an intrapulmonary tumor that had not in-

${ }^{1}$ Department of Chest Surgery, Toho University Medical Center Omori Hospital, Tokyo, ${ }^{2}$ Department of Respiratory Medicine, Toho University Medical Center Omori Hospital, Tokyo and ${ }^{3}$ Department of Surgical Pathology, Toho University Medical Center Omori Hospital, Tokyo Received for publication December 5, 2009; Accepted for publication February 28, 2010 Correspondence to Dr. Yoshinobu Hata, yoshinobuhata@hotmail.com 

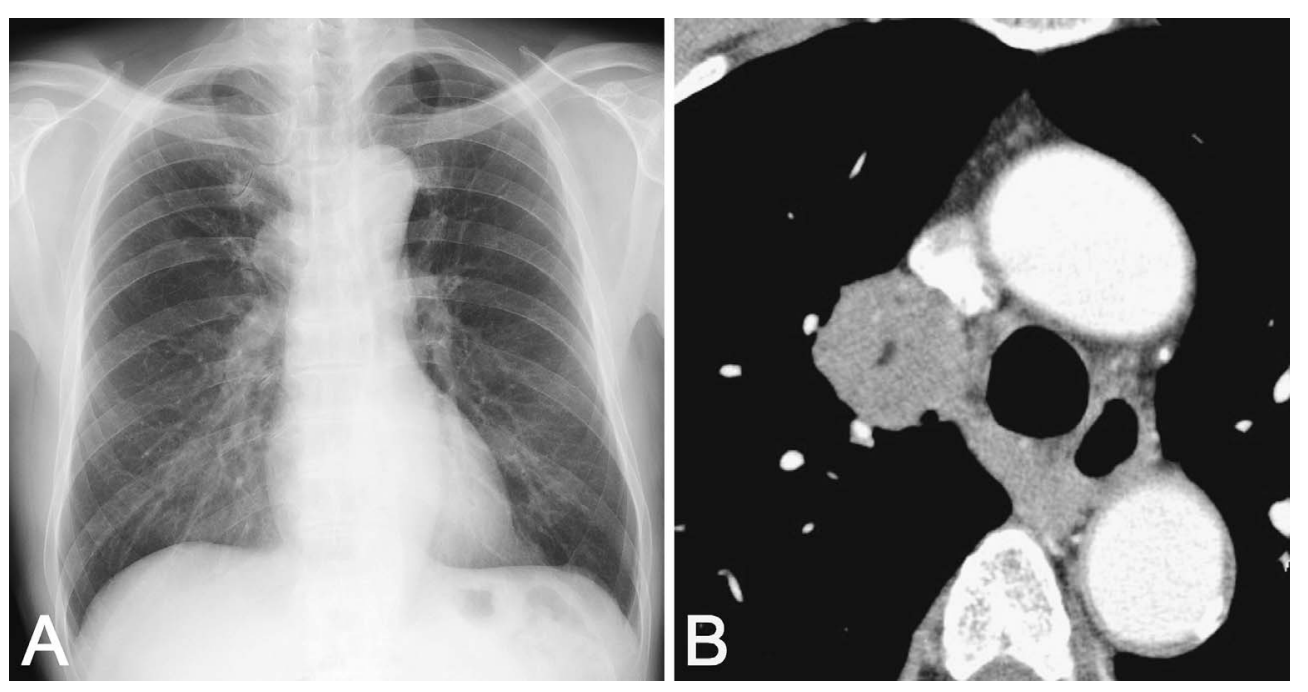

Figure 1. Chest radiograph showing a right hilar mass (A). Chest CT-scan showing a small fatdensity area in the right hilar mass adjacent to the trachea and superior vena cava $(B)$.

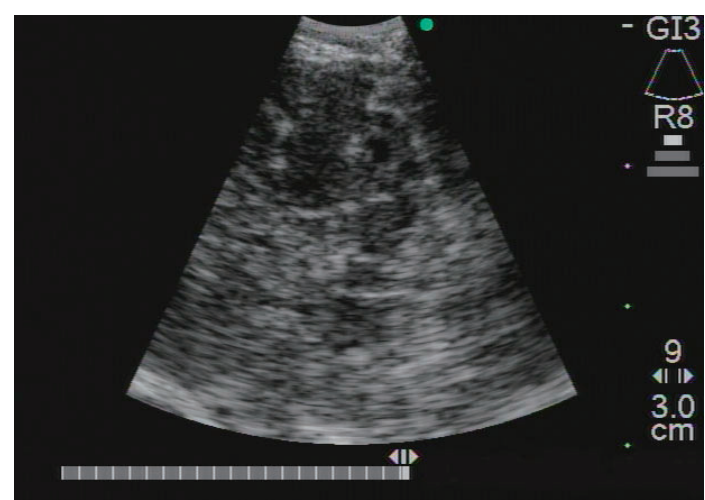

Figure 2. Endobronchial ultrasound (EBUS) images revealing a heterogeneous low-echoic mass consisting of highechoic dividing lines and coarse spots inside, which showed a smooth sliding movement during respiration.

vaded the trachea or superior vena cava. EBUS-TBNA was performed twice under real-time guidance with a 22-gauge needle (NA-201SX-4022, Olympus). Minute fragments were obtained through each pass with 5 strokes, after removing the inner canula of the needle which minimized contamination. Histological examination revealed that the small tumor sample thus obtained was entirely composed of mature cartilaginous tissue in which chondrocytes showed irregularity in size, shape and arrangement. In addition, empty lacunae were prominent, in contrast to cartilaginous tissue in normal bronchi. These findings suggested the presence of hamartoma at the site of biopsy (Fig. 3). The patient requested follow-up without surgical intervention. The tumor was found not to have enlarged after one year.

\section{Discussion}

Although CP-EBUS was developed mainly for pathological examination for lymph node staging, it is also valuable for diagnostic imaging (2). The linear ultrasound images are

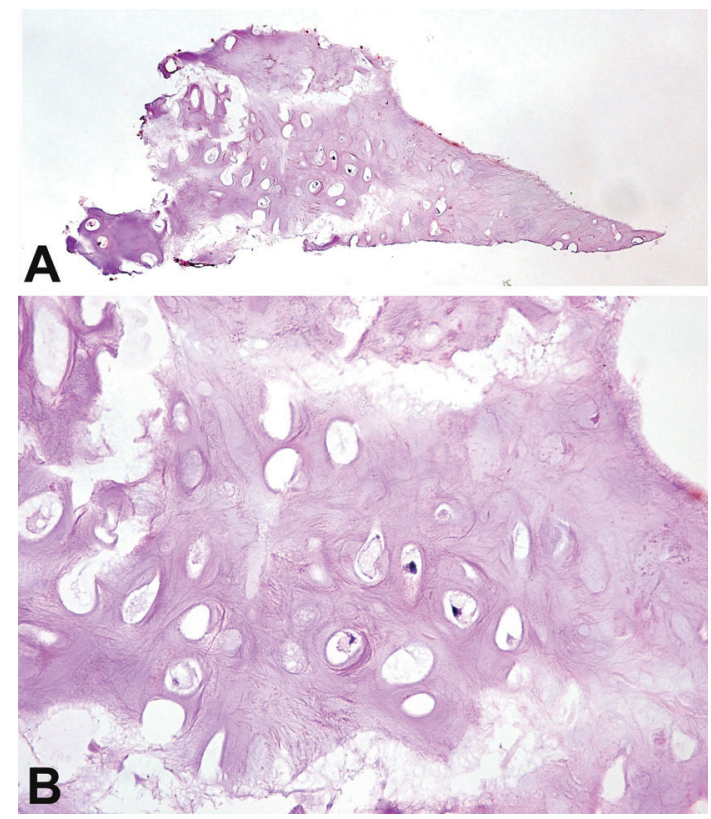

Figure 3. Microphotograph (Hematoxylin and Eosin staining, A: $\times 100$, B: $\times 400$ ) showing mature cartilaginous tissue with chondrocytes which are irregular in size, shape and arrangement, as well as empty lacunae.

easy to interpret and provide useful information on the area around the tracheal and bronchial tree. With the ability to observe smooth sliding movements of lesions during respiration, mediastinal tumor or malignant intrapulmonary tumor invading to the mediastinum could be ruled out noninvasively. Evaluation of the sensitivity and specificity of this respiratory dynamic approach will require validation in future studies.

Lung tumors located adjacent to the trachea or bronchus, which are sometimes difficult to reach using conventional bronchoscopic biopsy procedures or with CT-guided transthoracic needle aspiration biopsy, are a good indication for applying CP-EBUS-TBNA. Yasufuku et al reported that 
lung tumors that are located adjacent to the airway within the reach of CP-EBUS can be diagnosed with EBUSTBNA (1).

Pulmonary hamartoma is the most common benign tumor of the lung, with an incidence of $0.025 \%-0.32 \%$ (3). It consists of a mixture of undifferentiated mesenchymal cells, cartilage, fat, and cleft-like spaces lined by simple cuboidal or ciliated epithelium. Specific chromosomal aberrations were reported, suggesting that pulmonary hamartoma is a benign true neoplasm rather than a developmental anomaly (4). Intranodular fat is reported as a diagnostic CT finding, with a frequency of around 50\% (5). Although "popcorn" calcification is a well-known finding of pulmonary hamartoma (6), typical popcorn calcification is reported at a low frequency of only $5 \%$ to $15 \%(7,8)$. De Cicco et al reported that pulmonary hamartoma consists mainly of chondroid tissue (median 85\%; range, $0 \%$ to $85 \%$ ) with varying proportions of fibromyxoid tissue (median 14\%; range, 5\% to $80 \%$ ), fat (median $10 \%$; range, $1 \%$ to $45 \%$ ), bronchialtype epithelium (median $8 \%$; range, $2 \%$ to $10 \%$ ), and smooth muscle (median 2\%; range, $0 \%$ to $15 \%$ ), with vari- able lymphoid cell infiltrates observed in as many as $80 \%$ of cases, always accounting for $5 \%$ or less of the specimens (median 2\%; range, $2 \%$ to $5 \%$ ) (9).

Malignant conversion in pulmonary hamartoma (10) is exceedingly rare and quite controversial. Surgical intervention is recommended if the mass is larger than $2.5 \mathrm{~cm}$ (11). The cut-off value of " $2.5 \mathrm{~cm}$ " was derived from the CT criteria of Siegelman et al: a diameter of $2.5 \mathrm{~cm}$ or less, a smooth edge, and focal collections of fat or fat alternating with areas of calcification. They reported that no case of cancer or metastatic disease fulfilled these criteria (5). A mass larger than $2.5 \mathrm{~cm}$, with a definite histological diagnosis but without a tendency to enlarge can be kept under observation without resorting to surgery. The growth rate is usually very low, increasing by an average of $3.2+/-2.6 \mathrm{~mm}$ per year (12).

The need for diagnosing solitary pulmonary nodules is increasing with the increased use of CT. We conclude that CPEBUS is useful both for imaging and pathological examination in this context.

\section{References}

1. Yasufuku K, Nakajima T, Chiyo M, Sekine Y, Shibuya K, Fujisawa T. Endobronchial ultrasonography: current status and future directions. J Thorac Oncol 2: 970-979, 2007.

2. Aumiller J, Herth FJ, Krasnik M, Eberhardt R. Endobronchial ultrasound for detecting central pulmonary emboli: a pilot study. Respiration 77: 298-302, 2009.

3. Gjevre JA, Myers JL, Prakash UB. Pulmonary hamartomas. Mayo Clin Proc 71: 14-20, 1996.

4. Kazmierczak B, Meyer-Bolte K, Tran KH, et al. A high frequency of tumors with rearrangements of genes of the HMGI(Y) family in a series of 191 pulmonary chondroid hamartomas. Genes Chromosomes Cancer 26: 125-133, 1999.

5. Siegelman SS, Khouri NF, Scott WW Jr, et al. Pulmonary hamartoma: CT findings. Radiology 160: 313-317, 1986.

6. Park CM, Goo JM. Images in clinical medicine. "Popcorn" calcifications in a pulmonary chondroid hamartoma. $\mathrm{N}$ Engl J Med 360: e17, 2009.

7. Ledor K, Fish B, Chaise L, Ledor S. CT diagnosis of pulmonary hamartomas. J Comput Tomogr 5: 343-344, 1981.

8. Guo W, Zhao YP, Jiang YG, Wang RW, Ma Z. Surgical treatment and outcome of pulmonary hamartoma: a retrospective study of 20-year experience. J Exp Clin Cancer Res 27: 8, 2008.

9. De Cicco C, Bellomi M, Bartolomei M, et al. Imaging of lung hamartomas by multidetector computed tomography and positron emission tomography. Ann Thorac Surg 86: 1769-1772, 2008.

10. Rossi G, Cavazza A, Valli R, et al. Atypical lipomatous tumour (lipoma-like well-differentiated liposarcoma) arising in a pulmonary hamartoma and clinically presenting with pneumothorax. Lung Cancer 39: 103-106, 2003.

11. de Rooij PD, Meijer S, Calame J, Golding RP, van Mourik JC, Stam J. Solitary hamartoma of the lung: is thoracotomy still mandatory? Neth J Surg 40: 145-148, 1988.

12. Hansen CP, Holtveg H, Francis D, Rasch L, Bertelsen S. Pulmonary hamartoma. J Thorac Cardiovasc Surg 104: 674-678, 1992.

(C) 2010 The Japanese Society of Internal Medicine http://www.naika.or.jp/imindex.html 